\title{
Relationship between synaptic and functional connections of a local cortical network model Katsunori Kitano*1, Kazuhiro Yamada ${ }^{2}$ and Tomoki Fukai ${ }^{3}$
}

\author{
Address: ${ }^{1}$ Department of Human and Computer Intelligence, Ritsumeikan University, Kusatsu, Japan, ${ }^{2}$ Graduate School of Science and \\ Engineering, Ritsumeikan University, Kusatsu, Japan and ${ }^{3}$ Laboratory for Neural Circuit Theory, RIKEN Brain Science Institute, Wako, Japan \\ Email: Katsunori Kitano* - kitano@ci.ritsumei.ac.jp \\ * Corresponding author
}

from Sixteenth Annual Computational Neuroscience Meeting: CNS*2007

Toronto, Canada. 7-12 July 2007

Published: 6 July 2007

BMC Neuroscience 2007, 8(Suppl 2):P69 doi:10.1 I86/147|-2202-8-S2-P69

(C) 2007 Kitano et al; licensee BioMed Central Ltd.

Synaptic connectivity must have a significant impact on the dynamical behavior of neuronal networks and neural information processing. However, not much is known about the relation between neuronal activity and synaptic connectivity except for networks with simple connectivity, such as all-to-all or random connections. To clarify the issue, we first constructed a computational model of a local cortical network with realistic neuron models and systematically varied synaptic connections in a paradigm of the "small-world" network. Numerical simulations with the model showed spike activities depended on the network topology as well as the strengths of synaptic connections. In particular, the degree of pairwise spike synchrony, spatially distributed, characterizes the network connectivity. Therefore, we next tried to estimate the underlying synaptic structure based on spike data evoked by the model network. We defined functional connections with a measure of the pairwise spike synchrony, the coherence index, to discuss how the topology of the functional connections is related to that of synaptic connections. 